\title{
NMR, ESR, NQR and IR Studies of Paramagnetic Macrocyclic Complexes of first Transition Series Metal lons Exhibiting MLCT Phenomenon (A DFT Application) Part: II. Bis (1, 10-Phenanthroline) Complexes
}

\author{
M. L. SEHGAL ${ }^{1}$ and MD. IRSHAD AHMAD ${ }^{2 *}$ \\ ${ }^{1}$ Fmr. Head Chemistry, D.A.V. College, Jalandhar-144008, India. \\ 2Department of Biochemistry, Faculty of Life Sciences, Aligarh Muslim University, \\ Aligarh, 202002, Uttar Pradesh, India. \\ *Corresponding author E-mail: irshadahmad.bio@gmail.com
}

http://dx.doi.org/10.13005/ojc/330416

(Received: May 28, 2017; Accepted: June 29, 2017)

\begin{abstract}
D F T implemented in ADF.2012.01 was used to study the structures of 8 macrocyclic paramagnetic four coordinate $\mathrm{D}_{2 \mathrm{~d}}$ complexes:[Phen $\mathrm{M}^{2+}\{\mathrm{M}=\mathrm{Mn}$ (II), $\mathrm{Fe}(\mathrm{II}), \mathrm{Co}(\mathrm{II}), \mathrm{Ni}(\mathrm{II}), \mathrm{Cu}(\mathrm{II})\} ; \mathrm{Phe}$ $\left.\mathrm{n}_{2} \mathrm{M}\right]^{3+}\{\mathrm{M}=\mathrm{Ti}(\mathrm{III}) \mathrm{V}(\mathrm{III}), \mathrm{Cr}(\mathrm{III})\}$ (Phen=1,10-phenanthroline) by applying four spectral techniques. After pre-optimization of complexes, the software was run by using Single Point, LDA or GGA, Default, Relativity, Spin Orbit, ZORA, Unrestricted, None, Collinear, Nosym using TZP or TZ2P Basis sets in ESR/EPR/EFG/ZFS Program to obtain ESR parameters: $\mathrm{g}_{11}, \mathrm{~g}_{22}, \mathrm{~g}_{33}, \mathrm{~g}_{\mathrm{iso}}, \mathrm{a}_{11}, \mathrm{a}_{22}, \mathrm{a}_{33}, \mathrm{~A}_{\text {ten }}$. More ESR parameters $\left(g_{n} \cdot A_{\text {ten }}, Z F S\right)$ and NQR parameters $\left\{\eta, q_{11}, q_{22}, q_{33}, N Q C C\right\}$ were obtained on replacing Spin Orbit by scalar command in a new ADF Input. The "NMR Program" with Single Point, LDA, Default, None, Collinear, Nosym using DZ or TPZ Basis sets leaving Unrestricted command blank gave the Shielding Constants $\left(\sigma \mathrm{M}, \sigma^{13} \mathrm{C}, \sigma^{17} \mathrm{O}\right)$, Chemical Shifts $\left(\delta \mathrm{M}, \delta^{13} \mathrm{C}, \delta^{17} \mathrm{O}\right), 2$ diamagnetic, 4 paramagnetic and 4 spin orbit contributing terms in the $\sigma$ values of the constituents. The software was, then, run with Frequencies to obtain IR frequencies of normal modes of each of 129 fundamental vibration bands of the complexes. Importance of the study would lie in the fact that: (i) 5 parameter: $\sigma, \delta, \eta, g_{n} \cdot A_{\text {ten, }}$ NQCC of ${ }^{14} \mathrm{~N} ; 4$ parameters: $\sigma, \delta, g_{n}$. $A_{\text {ten, }} \eta$ of ${ }^{13} \mathrm{C}$ and 3 parameters: $\sigma, \delta, g . A_{\text {ten }}$ of ${ }^{1} \mathrm{H}$ corroborated to infer that in all these complexes, the $24 \mathrm{C}$ were of 6 types; the $16 \mathrm{H}$ of 4 types and all the $4 \mathrm{~N}$ were spatially of the same type(ii) it confirmed MLCT phenomenon by NMR(iii) calculated another parameter of each of NMR $\left(\mathrm{H}^{\wedge}\right)$ and $\operatorname{ESR}\left\{\Delta \mathrm{E}_{\mathrm{h}\}}\right\}$ and two of NQR $\{\eta$, Laplace equation $\}$ (iv) classified129 bands into vibration symmetries and IR activities and (iv) gave thermal parameters of the complexes.
\end{abstract}

Keywords: Chemical Shift, Total NMR Shielding Tensor, Effective Spin Hamiltonian, Nuclear Quadrupole Coupling Constant, Asymmetric Coefficient . 


\section{INTRODUCTION}

Aromatic heterocyclic compounds such as 1, 10-phenanthroline (Phen) and 2, 2-bipyridine (Bipy) form an important class of compounds in which the $\Pi$ and $n$ electrons can form chargetransfer complexes ${ }^{1,2}$ where the electronic charge of their complexes having filled $\Pi$ - molecular orbitals of energy comparable to the metal orbitals get transferred into the empty molecular orbitals with energy nearly equal to the ligand orbitals. They absorb in the visible region to exhibit intense color which is termed as Metal to Ligand Charge Transfer (MLCT) phenomenon. Unlike 2,2'-bipyridine, the1,10-phenanthroline does not have the same conformational flexibility and it would bind metal ions more strongly ${ }^{3,4}$. This enantioselective interaction of 1, 10-phenanthroline complexes of iron (II), Co (III), $\mathrm{Zn}$ (II) is used as a structural probe and mediators of DNA cleavage reactions ${ }^{5-7}$. Homoleptic bis $(1,10-$ phenanthroline) transition metal ions complexes $\left[\mathrm{Phen}_{2} \mathrm{M}\right]^{2+,}{ }^{3+}$ were studied comparatively to a lesser extent both experimentally and by Density Functional Theory(DFT). Single crystal structures of bis [chloro bis (1,10-phenanthroline- $\mathrm{N}, \mathrm{N}^{\prime}$ ) (thiourea -S) nickel(II)] chloride nitrate was studied by L. Suescun and his coworkers ${ }^{8}$. Structure and magnetic properties of di- $\mu$-hydroxo-bis [bis $(1,10$ phenanthroline) chromium(III)] iodide tetrahydrate were studied by Raymond Scaringe and Phritu Singh ${ }^{9}$. O. Yesilel, H. Olmez studied spectrothermal properties of 1, 10-phenanthroline complexes along with $\mathrm{X}$-rays single crystal structure and photo luminance properties of $\mathrm{Co}$ (II), $\mathrm{NI}$ (II), $\mathrm{Cu}$ (II) and Cd (II) orotates with UV-Vis; and $\mathrm{FTIR}^{10}$.V. Chis et al ${ }^{11}$ studied Spin-state cross-over in Fe $\left(\right.$ Phen $_{2}(\mathrm{NCS})_{2}$ by DFT. Ozel Aysen E. and coworkers ${ }^{12}$ studied vibration spectra of copper(II)2,2'-bipyridine and 1,10-phenanthroline complexes by applying $\mathrm{DFT}^{12}$. DFT IR, UV and Mossbauer of 2, 2'-bipyridine and 1, 10-phenanthroline complexes of iron (II) was studied by Morigakii, Milton K. et $\mathrm{al}^{13}$ with the help of DFT.H.Z. Chiniforoshan et $\mathrm{al}^{14}$ studied the structures of complexes of $\mathrm{Co}$ (III) and Zn (II) with 2,2'-bipyridine and 1,10-phenanthroline by applying DFT. Sumit Sanotra et al applied DFT to study X-ray crystal structure and photo luminance properties of $[\mathrm{Cd}$ $\left(\mathrm{NO}_{3}\right)_{2}(\text { phen })_{2}{ }^{15}$. Euan $\mathrm{K}$. Bre chin and coworkers ${ }^{16}$ studied UV,ESR and magnetic properties of $\mathrm{Cr}(\mathrm{III})$, $\mathrm{Fe}$ (II).N.S. Panina and coworkers ${ }^{17}$ studied stabilities of high and low spin isomers and $\mathrm{X}$-ray structures of Ni(II) complexes. Zn Bz Li and Xiao yuan studied Raman spectra of bis- and tris (1, 10-phenanthroline) manganese (II) complexes ${ }^{18}$.

\section{Need for the study}

- A limited experimental research had been carried out in the calculation of NMR, ESR and NQR parameters of these 8 macro cyclic paramagnetic 1,10-phenanthroline complexes: $\left[\mathrm{Phen}_{2} \mathrm{M}\right]^{2+}\{\mathrm{M}=\mathrm{Mn}$ (II), $\mathrm{Fe}(\mathrm{II})$ $\mathrm{Co}(\mathrm{II}), \mathrm{Ni}(\mathrm{II}), \mathrm{Cu}$ (II)\}; [Phen $\left.\mathrm{M}_{2}\right]^{3+}\{$ $\mathrm{M}=\mathrm{Ti}(\mathrm{III}) \mathrm{V}(\mathrm{III}), \mathrm{Cr}(\mathrm{III})\}$ as accurate computations of parameters of these techniques ${ }^{19-23} \mathrm{had}$ become possible by $\mathrm{DFT}^{24,25}$ only recently .

$\mathrm{v}_{(\mathrm{CN})}$ would be lowered on coordination of $\mathrm{N}$ to a metal ion while the transfer of electronic charge from molecular orbitals mainly with metal character to those having the ligand character should increase $v_{(\mathrm{CN})}$. Since $v_{(\mathrm{CN})}$ was metal sensitive only to a limited extent, it would be difficult to assign exact value to $\mathrm{v}_{\text {(CN) }}$ as many 1, 10-phenanthroline vibrations also appeared in the same region ${ }^{26}$. Also the $\Pi \rightarrow \Pi^{*}$ transition of the ligand and MLCT in some complexes ${ }^{27}$ would absorb in the same region $^{19}$, the reflectance spectral technique might not help. Thus the NMR technique was tried which also enabled us to calculate the Spin Hamiltonian $\left(\mathrm{H}^{\wedge}\right)$ values of these complexes

\section{Methodology}

Use of ADF (Amsterdam Density Functional) software to calculate various NMR ${ }^{(29-34)}, \mathrm{IR}^{(28)}$, ESR ${ }^{(35-39)}$ and NQR ${ }^{(35-39)}$-parameters had, already, been reported.

\section{RESULTS}

Tables: 1-3 represented energies of metals, their $g_{n}$ and I values, thermal and optimization parameters of complexes. Table 4 gave $\sigma$ and $\delta$ values of ${ }^{14} \mathrm{~N},{ }^{13} \mathrm{C}$ and ${ }^{1} \mathrm{H}$ of the free 1,10 phenanthroline. Table 5 contained $\sigma$ and $\delta$ values of $\mathrm{M}^{+n},{ }^{14} \mathrm{~N},{ }^{13} \mathrm{C}$ and ${ }^{1} \mathrm{H}$ of complexes. Table 6 contained contributions of 2 diamagnetic, 4 paramagnetic and 4 spin orbit terms (10 in all) in $\sigma$ of $\mathrm{M}^{\mathrm{n}+14} \mathrm{~N},{ }^{13} \mathrm{C},{ }^{1} \mathrm{H}$ of complexes .Table: 7 gave ESR and NQR parameter of the various types of $\mathrm{H}, \mathrm{C}$ and $\mathrm{N}$ in complexes. 
Table 8 gave some more ESR and NQR parameters of complexes. Table 9 contained contributions from five factors into $H$ spin and $\Delta \mathrm{E}_{\mathrm{h} f}$ values. Table 10 designated IR-active bands in the complexes. Table 11 gave $\sigma^{14} \mathrm{~N}$ values of the uncoordinated ligand and the complexes.

Figure 1 gave ADF numbers of the 8 bis (1, 10-phenanthroline) complexes

\section{DISCUSSION}

Each one of the eight 1, 10-phenanthroline complexes contained 45 atoms: $4 \mathrm{~N}, 16 \mathrm{H}, 24 \mathrm{C}$ and a different transition metal ion.

Relations used to calculate NMR parameters ${ }^{40}$

- $\quad \sigma \mathrm{M}^{\mathrm{n}+}, \sigma^{1} \mathrm{H}, \sigma^{13} \mathrm{C}$ and $\sigma^{14} \mathrm{~N}$ were equal to the sum of values of 2 diamagnetic, 4

Table: 1. Energies $\left(\mathrm{kJmol}^{-1}\right)$ of First Transition Metals

\begin{tabular}{lllllll}
\hline $\mathbf{M}$ & $\begin{array}{l}\text { Sum of } \\
\text { orbital } \\
\text { energies }\end{array}$ & $\begin{array}{l}\text { Total } \\
\text { energy }\end{array}$ & $\begin{array}{l}\text { Kinetic } \\
\text { energy }\end{array}$ & $\begin{array}{l}\text { Nuclear } \\
\text { attraction } \\
\text { energy }\end{array}$ & $\begin{array}{l}\text { Electron } \\
\text { repulsion } \\
\text { energy }\end{array}$ & $\begin{array}{l}\text { Exchange } \\
\text { energy }\end{array}$ \\
\hline $\mathrm{Ti}$ & -49034.160 & -82193.590 & 83068.461 & -195833.367 & 34453.298 & -3879.077 \\
$\mathrm{~V}$ & -54453.277 & -91394.073 & 92457.572 & -218055.74 & 38338.78 & -4184.954 \\
$\mathrm{Cr}$ & -60139.891 & -101188.38 & 102462.98 & -241698.34 & 42549.246 & -4503.221 \\
$\mathrm{Mn}$ & -18580.3096 & -31364.4439 & 31791.632 & -75034.4123 & 13237.036 & -1358.702 \\
$\mathrm{Fe}$ & -20333.522 & -344593.341 & 349652.94 & -82578.841 & 14339.914 & -1455.918 \\
$\mathrm{Co}$ & -78854.221 & -134234.27 & 136350.81 & -346177.79 & 57226.702 & -5539.960 \\
$\mathrm{Ni}$ & -85650.855 & -146522.795 & 148997.80 & -352449.098 & 62843.648 & -8809.705 \\
$\mathrm{Cu}$ & -9309.418 & -159479.16 & 162355.29 & -384859.801 & 69353.856 & -6328.501 \\
\hline
\end{tabular}

Table: 2. Optimization Parameters $\left(\mathrm{kJmol}^{-1}\right),\left[\mathrm{M} \mathrm{g}_{\mathrm{n}}\right],\left\{\mathrm{M}_{1}\right\}$ and $\left(\mathrm{M}_{\mathrm{S}}\right)$ of $\left[\mathrm{Phen}_{2} \mathrm{M}^{\mathrm{n}+}{ }^{+}\right.$Complexes

\begin{tabular}{|c|c|c|c|c|}
\hline$M^{n+}\left(D_{2 d}\right)$ & $\begin{array}{l}{\left[M g_{n}\right]} \\
\left\{M_{1}\right\} \\
\left(M_{s}\right)\end{array}$ & $\begin{array}{l}\text { Total } \\
\text { bonding } \\
\text { energy }{ }^{\star *}\end{array}$ & 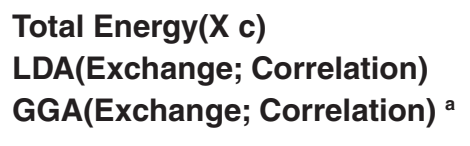 & Nucleus \\
\hline $\mathrm{Ti}(\mathrm{III})^{\mathrm{a}}$ & $\begin{array}{l}{[-0.315392]\{2.5\}} \\
(0.5)\end{array}$ & -25980.97 & $\begin{array}{l}-511912.56 \\
-442448.48,-34582.43 \\
-52792.02,17910.37\end{array}$ & ${ }^{47} \mathrm{Ti}$ \\
\hline V(III) & $\begin{array}{l}{[1.4710588]\{3.5\}} \\
(1.0)\end{array}$ & -28816.33 & $\begin{array}{l}-484494.18 \\
-449671.64,-34822.54\end{array}$ & ${ }^{51} \mathrm{~V}$ \\
\hline $\mathrm{Cr}(\mathrm{III})^{\mathrm{a}}$ & $\begin{array}{l}{[-0.316360]\{1.5\}} \\
(1.5)\end{array}$ & -26138.48 & $\begin{array}{l}-529520.63 \\
-458827.75,-35164.23 \\
-53626.90,18098.25\end{array}$ & ${ }^{56} \mathrm{Cr}$ \\
\hline $\mathrm{Mn}(\mathrm{II})$ & $\begin{array}{l}{[1.387488]\{2.5\}} \\
(2.5)\end{array}$ & -30145.34 & $\begin{array}{l}-501580.82 \\
-466197.13,-35383.70\end{array}$ & ${ }^{55} \mathrm{Mn}$ \\
\hline $\mathrm{Fe}(\mathrm{II})$ & $\begin{array}{l}{[0.181246]\{0.5\}} \\
(2.0)\end{array}$ & -29988.39 & $\begin{array}{l}-510676.41 \\
-475004.40,-35672.01\end{array}$ & ${ }^{57} \mathrm{Fe}$ \\
\hline $\mathrm{Co}(\mathrm{II})$ & $\begin{array}{l}{[1.322000]\{3.5\}} \\
(1.5)\end{array}$ & -29955.67 & $\begin{array}{l}-520137.94 \\
-484172.74,-35965.19\end{array}$ & ${ }^{59} \mathrm{Co}$ \\
\hline $\mathrm{Ni}(\mathrm{II})$ & $\begin{array}{l}{[-0.500133]\{1.5\}} \\
(1.0)\end{array}$ & -29673.45 & $\begin{array}{l}-530709.68 \\
-494411.25,-36298.43\end{array}$ & ${ }^{61} \mathrm{Ni}$ \\
\hline $\mathrm{Cu}(\mathrm{II})$ & $\begin{array}{l}{[-1.4821933]\{1.5\}} \\
(0.5)\end{array}$ & -29439.21 & $\begin{array}{l}-540776.87 \\
-504181.31,-36595.56\end{array}$ & ${ }^{63} \mathrm{Cu}$ \\
\hline
\end{tabular}

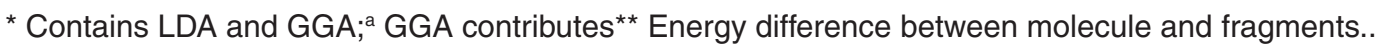


paramagnetic and 4 spin orbit terms of $\mathrm{M}^{\mathrm{n}+},{ }^{1} \mathrm{H},{ }^{13} \mathrm{C}$ and ${ }^{14} \mathrm{~N}$ respectively.

- $\quad(\sigma)$ and $(\delta)$ of ${ }^{1} \mathrm{H},{ }^{13} \mathrm{C}$ and ${ }^{14} \mathrm{~N}$ were related as follows:

$\delta^{1} H=31.7-\sigma^{1} H$

$\delta^{13} \mathrm{C}=181.1-\sigma^{13} \mathrm{C}$

$$
\begin{array}{r}
\sigma M=-\delta M I \\
\sigma^{17} O=-\delta^{14} N \mid
\end{array}
$$

Relations used to Calculate ESR parameters ${ }^{35-37}$ Effective Spin Hamiltonian $\left(\mathbf{H}^{\wedge}\right)^{35-37}$

No doubt, three relations ${ }^{35-39}$ were needed to calculate $\mathrm{H}^{\wedge}$ but all the 8 complexes included in the present study were axially asymmetric having the same two $g$ values $(g \perp)$ while the third $g$ of different value and was represented as $g_{\|}$. Correspondingly, two same a values $(\mathrm{a} \perp$ ) while the third a of different value was named as $a_{\|}$, only the following relation would suffice:

$$
\begin{array}{r}
H^{\wedge}=\beta_{e}\left[g_{\|} \cdot H_{\|} \cdot S+g \perp(2 H \perp . S)\right]+\left[a_{\|} \cdot S . I\right. \\
+a \perp(2 S . I)]+Q[I-1 / 3 I(I+1)]+D\left\{S_{z}^{2}-S(S+1) / 3\right\}- \\
{\left[g_{n} \cdot \beta_{n} \cdot H_{0} . I\right]}
\end{array}
$$

Five factors: $g$ factor, a factor, $Q$ factor, Zero Field Splitting (ZFS) factor ${ }^{41,42}$ and interaction of nuclear magnetic moment with external magnetic

\begin{tabular}{|c|c|c|c|c|c|c|c|c|c|c|c|c|c|}
\hline \multirow[t]{3}{*}[M]{$^{n+}$} & \multirow{3}{*}{$\begin{array}{l}\text { Zero } \\
\text { Point } \\
\text { Volume } \\
\text { Energy } \\
(\mathrm{e} \text { V) }\end{array}$} & & & & & \multicolumn{8}{|c|}{ Thermal Parameters } \\
\hline & & \multicolumn{4}{|c|}{ Entropy (cal mol-1 $\mathrm{K}^{-1}$ ) } & \multicolumn{4}{|c|}{$\begin{array}{l}\text { Internal Energy } \\
\left(\text { Kcal mol}^{-1}\right)\end{array}$} & \multicolumn{4}{|c|}{$\begin{array}{l}\text { Constant } \\
\left.\text { Capacity (Kcal mol-1 } \mathrm{K}^{-1}\right)\end{array}$} \\
\hline & & Trans. & Rot. & Vib. & Total & Trans. & Rot. & Vib. & Total & Trans. & Rot. & Vib. & Total \\
\hline (III) & 9.2711 & 43.91 & 33.1 & 54.3 & 131.26 & 0.889 & 0.889 & 223.3 & 225.1 & 2.981 & 2.981 & 73.88 & 79.84 \\
\hline V(III) & 9.246 & 43.93 & -do- & 49.0 & 126.00 & -do- & - do- & 222.3 & 224.1 & -do- & -do- & 74.43 & 80.39 \\
\hline $\mathrm{Cr}(\mathrm{III})$ & 9.2647 & 43.94 & -do- & 51.2 & 128.21 & -do- & -do- & 222.9 & 224.7 & -do- & -do- & 74.03 & 80.0 \\
\hline $\mathrm{Mn}(\mathrm{II})$ & 9.256 & 43.96 & -do- & 56.4 & 133.38 & -do- & -do- & 223.5 & 225.2 & -do- & -do- & 77.97 & 83.93 \\
\hline $\mathrm{Fe}(\mathrm{II})$ & 9.277 & 43.97 & -do- & 70.4 & 147.44 & -do- & -do- & 225.4 & 227.2 & -do- & -do- & 83.66 & 89.62 \\
\hline $\mathrm{Co}(\mathrm{II})$ & 9.244 & 43.99 & -do- & 63.3 & 140.34 & -do- & -do- & 223.8 & 225.6 & -do- & -do- & 80.3 & 86.3 \\
\hline $\mathrm{Vi}(I I)$ & 9.222 & 43.98 & -do- & 60.7 & 137.74 & -do- & -do- & 223.0 & 224.7 & -do- & -do- & 78.68 & 84.64 \\
\hline $\mathrm{Cu}(\mathrm{II})$ & 9.294 & 44.02 & -do- & 64.1 & 141.21 & -do- & -do- & 225.2 & 227.0 & -do- & -do- & 81.34 & 87.30 \\
\hline
\end{tabular}
field, i.e. I factor would contribute in the total value of Spin Hamiltonian $\left(\mathrm{H}^{\wedge}\right) . \mathrm{S}_{z}$ representing spin angular

\begin{tabular}{|c|c|c|c|c|c|c|}
\hline Ligand $\left[\mathrm{C}_{2 \mathrm{v}}\right]$ & $\delta \mathbf{N}^{\mathrm{b}}{ }_{(3)}$ & $\sigma \mathbf{N}$ & $\delta \mathbf{C}^{\mathbf{c}}{ }_{(2)}$ & $\sigma \mathbf{C}$ & $\delta \mathbf{H}_{(1)}^{\mathrm{d}}$ & $\sigma \mathbf{H}$ \\
\hline $\begin{array}{l}\text { 1, 10-phenan- } \\
\text { throline }\end{array}$ & $\begin{array}{l}(6,16) \\
138.4\end{array}$ & $\begin{array}{l}\mathrm{N}(6,16) \\
-138.4\end{array}$ & $\begin{array}{l}(1,11) 133.38 \\
(2,12) 107.25 \\
(3,13) 114,51 \\
(4,14) 104.94 \\
(5,15) 144.56 \\
(7,17) 114.14\end{array}$ & $\begin{array}{l}(1,11) 47.72 \\
(2,12) 73.85 \\
(3,13) 66.59 \\
(4,14) 76.16 \\
(5,15) 36.54 \\
(7,17) 66.96\end{array}$ & $\begin{array}{c}(8,18,21,22) 7.65 \\
(9,19) 7.23 \\
(10,20) 9.60\end{array}$ & $\begin{array}{l}(8,18,21,22) 24.06 \\
(9,19) 24.4(10,20) \\
22.1\end{array}$ \\
\hline
\end{tabular}
momentum was calculated as:

Table 3: Thermal Parameters of $\left[\text { Phen }_{2} \mathrm{M}\right]^{\mathrm{n}+}$ Complexes

Table: 4. $\sigma \mathrm{N}, \sigma \mathrm{H}, \delta \mathrm{N}$ and $\delta \mathrm{H}(\mathrm{ppm})$ of 1, 10- Phenanthroline ${ }^{\mathrm{a}}$

a.ADF Numbers in parentheses; b. standard zero; c. standard 181.1; d. standard 31.7; Apply Relation [1, 2,3 ] 
$S_{z}=S / S(S+1)^{0.5}$

\section{Hyperfine Coupling Energy ${ }^{41}$}

$\Delta \mathrm{E}_{\mathrm{hf}}=1 / 2\left[\mathrm{a}_{11}{ }^{2}+\mathrm{a}_{22}{ }^{2}+\mathrm{a}_{33}{ }^{2}\right]^{1 / 2}$

$\mathrm{H}^{\wedge}$ values were calculated both in terms of $\mathrm{MHz}$ as well as in joules mol'-

Their inter conversions were given as:

- One $\mathrm{MHz}=6.627^{*} 10^{-21} \mathrm{erg}=3.9903124^{*} 10^{-7}$ $\mathrm{kJ} \mathrm{mol}^{-1}$

- $1 \mathrm{~cm}^{-1}=0.0119626 \mathrm{~kJ} \mathrm{~mol}^{-1}=29979.2458$ $\mathrm{MHz}$

- $\quad$ For 8388.255 MHz in a 0.30T, the $\mathrm{g}$ value of the standard substance: 2, 2-diphenyl1-picrylhydrazyl (DPPH) was: $g_{D P P H}$ $2.00232^{43-45}$. So $g$ value of the complex $\left(g_{M}{ }^{n+}\right)$ and its frequency $\left(v_{M}{ }^{n+}\right)$ were related as follows:

$v_{m}{ }^{n+}=8388.255^{*} g_{M}{ }^{n+} / 2.00232$

The details of the various terms involved were discussed elsewhere ${ }^{28}$.
Relations used to Calculate NQR Parameters ${ }^{46,47}$ Asymmetry Coefficient (h) ${ }^{46,47}$

$\eta=q_{x x}-q_{y y} / q_{z z}$

( $\eta$ ) lies in between 0 -1.For axial symmetry, $\eta=0$. It was possible only when:

$q_{x x}=q_{y y} \neq q_{z z}$

Laplace Equation ${ }^{46,47}$

$q_{x x}+q_{y y}+q_{z z}=0$

Calculation of four NMR and NQR parameters Four more ESR and NQR parameters: $\mathrm{H}^{\wedge}, \Delta \mathrm{E}_{\mathrm{h} f}, \eta$, and Laplace equation which were calculated in addition to five $\operatorname{ESR}\left(g_{11}, g_{22}, g_{33}, g_{\text {iso }}\right.$; $\left.a_{11}, a_{22}, a_{33}, A_{\text {ten }}\right)$ and NQR parameters $\left(\eta ; q_{11}, q_{22}\right.$, $\mathrm{q}_{33}$; NQCC) parameters obtained from the software for 7 complexes excluding [Phen ${ }_{2} \mathrm{Fe}{ }^{2+}$ where ADF software did not work.

All the 7complexes possessed axial symmetry with (a) Two of three $g$ called $g \perp$ were of

Table: 5. $\sigma$ and $\delta$ values (ppm) of $\mathbf{M}^{+n}, \mathbf{N}, \mathbf{C}, \mathrm{H}$ in $\left[\right.$ Phen $_{2} \mathbf{M}^{\mathrm{n}+} \mathbf{W i t h}^{\mathbf{M}^{\mathrm{n}+}}{ }^{\text {Complexes }}{ }^{\mathrm{a}}$

\begin{tabular}{|c|c|c|c|c|c|c|c|c|c|c|c|c|}
\hline 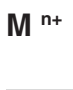 & $\begin{array}{l}\delta \mathbf{M}^{+n}[3] \\
\sigma \mathbf{M}^{+n}\end{array}$ & $\begin{array}{l}\delta \mathbf{N}^{[3]} \\
\sigma \mathbf{N}^{-}\end{array}$ & $\begin{array}{l}\delta \mathbf{H}^{[1]} \\
\sigma \mathbf{H}^{-1}\end{array}$ & $\begin{array}{l}\delta \mathbf{H}^{[1]} \\
\sigma \mathbf{H}^{-}\end{array}$ & $\begin{array}{l}\delta \mathbf{H}^{[1]} \\
\sigma \mathbf{H}^{-}\end{array}$ & $\begin{array}{l}\delta \mathbf{H}^{[1]} \\
\sigma \mathbf{H}^{-}\end{array}$ & $\begin{array}{l}\delta \mathbf{C}^{[2]} \\
\sigma \mathbf{C}\end{array}$ & $\begin{array}{l}\delta \mathbf{C}^{[2]} \\
\sigma \mathbf{C}\end{array}$ & $\begin{array}{l}\delta \mathbf{C}^{[2]} \\
\sigma \mathbf{C}\end{array}$ & $\begin{array}{l}\delta \mathbf{C}^{[2]} \\
\sigma \mathbf{C}\end{array}$ & $\begin{array}{l}\delta \mathbf{C}^{[2]} \\
\sigma \mathbf{C}\end{array}$ & $\begin{array}{c}\delta \mathbf{C}^{[2]} \\
\sigma \mathbf{C}\end{array}$ \\
\hline & 1 & $\begin{array}{l}7,17, \\
2939\end{array}$ & $\begin{array}{l}9,19 \\
31,41\end{array}$ & $\begin{array}{l}10,20 \\
32,42\end{array}$ & $\begin{array}{l}11,21 \\
33,43\end{array}$ & $\begin{array}{l}22,23 \\
44,45\end{array}$ & $\begin{array}{l}4,14 \\
26,36\end{array}$ & $\begin{array}{l}5,15 \\
27,37\end{array}$ & $\begin{array}{l}6,16 \\
28,38\end{array}$ & $\begin{array}{l}8,18 \\
30,40\end{array}$ & $\begin{array}{l}3,13, \\
25,35\end{array}$ & $\begin{array}{l}2,12 \\
24,34\end{array}$ \\
\hline $\mathrm{Ti}$ & 2119.95 & -316.5 & -57.62 & -47.07 & -43.9 & -48.17 & -230.3 & -158.00 & -9.11 & -236.62 & -241.10 & -235.5 \\
\hline (III) & -2119.95 & 316.50 & 89.32 & 78.77 & 75.56 & 79.87 & 411.35 & & 190.21 & 72 & 423.20 & \\
\hline V & 8217.00 & -296.3 & & -47.21 & -47.2 & -47.12 & -226.7 & & & & & \\
\hline (III) & -821 & 296.30 & 88.87 & 78.91 & & 78.82 & & & & & & \\
\hline$C_{1}$ & 3690 & -310.2 & -57.98 & -46.54 & -42.7 & -48.49 & -228.4 & 40 & -24.41 & .00 & 9.40 & -2 \\
\hline (III) & -3690.89 & 310.20 & 89.68 & 78.24 & 73.40 & 80.19 & 409.46 & 46 & 205.51 & .13 & 420.54 & 392.80 \\
\hline $\mathrm{Mn}$ & 345.51 & -178.4 & -58.27 & -46.72 & -42.2 & -48.48 & -227.3 & .00 & -5.01 & 5.90 & 0.30 & -207.7 \\
\hline (II) & -345 & $17 \varepsilon$ & & 78.42 & 73.86 & & 408.4 & & 186.11 & & & \\
\hline $\mathrm{Fe}$ & 6885.75 & & & -46.65 & -41.7 & & & & & & 30 & \\
\hline (II) & -68 & 140.68 & 89.91 & 78.35 & 73.4 & 80 & 40 & & & & & \\
\hline Co & 14190.2 & -84.80 & -57.68 & -46.23 & -42.9 & $-47,8$ & -227.7 & -151.10 & -7.27 & -235.40 & -238.40 & -203.6 \\
\hline (II) & -14190.2 & 84.80 & 89.38 & 77.93 & 74.61 & 79.50 & 408.80 & 332.18 & 188.37 & 416.50 & 419.52 & 384.70 \\
\hline $\mathrm{Ni}$ & 11912.7 & 1.15 & -58.07 & -44.71 & -37.1 & -48.3 & -228.3 & -145.20 & 2.13 & -235.17 & -238.90 & -212.10 \\
\hline (II) & -11912.7 & -1.15 & 89.77 & 76.41 & 68.82 & 79.99 & 409.33 & 326.61 & 178.97 & 416.27 & 419.97 & 393.23 \\
\hline $\mathrm{Cu}$ & 3546.36 & 163.10 & -57.38 & -41.98 & -35.3 & -47.18 & -221.8 & -136.20 & 10.97 & -223.50 & -139.70 & -220.10 \\
\hline (II) & -3546.36 & -163.1 & 89.08 & 73.68 & 67.0 & 78.88 & 402.89 & 317.90 & 170.73 & 404.60 & 419.43 & 401.84 \\
\hline
\end{tabular}

a. ADF Numbers in parentheses [Fig .1] ; Apply Relation ${ }^{1,2,3}$ 
Table: 6. Diamagnetic, Paramagnetic \& Spin orbit contributions in $\sigma(\mathrm{ppm})$ of $\left[\mathrm{Phen}_{2} \mathrm{M}^{\mathrm{n}+}\right.$ Complexes $^{\text {a }}$

\begin{tabular}{|c|c|c|c|c|}
\hline$M^{n+}$ & $\sigma M^{+n}$ & $\begin{array}{l}\sigma \mathrm{N} \text { of each of the } 4 \mathrm{~N} \\
7,17,29,39\end{array}$ & $\begin{array}{l}\sigma \mathrm{H} \text { of } 4 \text { types of } \mathrm{H} \\
9,19,31,41,(10,20 \\
, 32,42), \\
\{11,21,33,43\},[22,23, \\
, 44,45]\end{array}$ & $\begin{array}{l}\sigma \mathrm{C} \text { of } 6 \text { types of } C \\
4,14,26,36,(5,15 \\
, 27,37), \\
\{6,16,28,38\},[8, \\
18,30,40] \\
((3,13,25,35)),\{\{2,12, \\
24,34\}\}\end{array}$ \\
\hline
\end{tabular}

\begin{tabular}{|c|c|c|c|c|c|c|c|c|c|c|c|c|}
\hline & Dia. & Para. & S.O. & Dia. & Para. & S.O. & Dia. & Para. & S.O. & Dia. & Para. & S.O. \\
\hline $\begin{array}{l}\mathrm{Ti} \\
\text { (III) }\end{array}$ & 1599.5 & -2510.16 & -1209.3 & 323.18 & -6.390 & -0.330 & $\begin{array}{l}28.163 \\
28.268 \\
28.593 \\
28.452\end{array}$ & $\begin{array}{l}61.255 \\
51.158 \\
48.098 \\
51.338\end{array}$ & $\begin{array}{l}-0.097 \\
-0.657 \\
-1.128 \\
0.076\end{array}$ & $\begin{array}{l}256.2 \\
257.2 \\
57.5 \\
255.2 \\
255.2 \\
255.4\end{array}$ & $\begin{array}{l}154.5 \\
86.22 \\
-27.45 \\
161.9 \\
167.5 \\
167.7\end{array}$ & $\begin{array}{l}0.65 \\
-4.25 \\
-39.8 \\
0.54 \\
0.38 \\
-6.56\end{array}$ \\
\hline $\begin{array}{l}\text { V } \\
\text { (III) }\end{array}$ & 1706.0 & -9199.86 & -723.1 & 322.88 & -3.785 & -22.80 & $\begin{array}{l}28.087 \\
28.257 \\
28.624 \\
28.376\end{array}$ & $\begin{array}{l}60.781 \\
51.259 \\
51.385 \\
50.424\end{array}$ & $\begin{array}{l}0.003 \\
-0.608 \\
-1.079 \\
0.020\end{array}$ & $\begin{array}{l}256.0 \\
257.2 \\
257.3 \\
255.0 \\
255.1 \\
254.8\end{array}$ & $\begin{array}{l}151.4 \\
85.53 \\
-33.20 \\
159.2 \\
163.2 \\
145.3\end{array}$ & $\begin{array}{l}0.36 \\
-2.21 \\
-22.8 \\
-0.08 \\
-1.05 \\
-19.6\end{array}$ \\
\hline $\begin{array}{l}\mathrm{Cr} \\
\text { (III) }\end{array}$ & 1812.2 & -4962.47 & -540.6 & 322.83 & 2.296 & -14.93 & $\begin{array}{l}28.067 \\
28.259 \\
28.658 \\
28.310\end{array}$ & $\begin{array}{l}61.561 \\
50.633 \\
45.956 \\
51.779\end{array}$ & $\begin{array}{c}0.048 \\
-0.655 \\
-1.212 \\
0.101\end{array}$ & $\begin{array}{l}255.8 \\
257.1 \\
257.3 \\
254.9 \\
255.0 \\
254.4\end{array}$ & $\begin{array}{l}153.8 \\
84.2 \\
-38.27 \\
160.3 \\
165.3 \\
147.9\end{array}$ & $\begin{array}{l}-0.11 \\
-0.86 \\
-13.5 \\
-0.05 \\
0.23 \\
-9.57\end{array}$ \\
\hline $\begin{array}{l}\mathrm{Mn} \\
\text { (II) }\end{array}$ & 1927.8 & -1905.32 & -368.0 & 322.72 & -14.21 & 28.770 & $\begin{array}{l}28.770 \\
29.041 \\
29.172\end{array}$ & $\begin{array}{l}60.914 \\
50.622 \\
48.012\end{array}$ & $\begin{array}{l}0.289 \\
-1.246 \\
-3.327\end{array}$ & $\begin{array}{l}255.6 \\
257.3 \\
256.6 \\
254.8 \\
254.8 \\
253.8\end{array}$ & $\begin{array}{c}156.2 \\
85.24 \\
-43.0 \\
161.8 \\
167.6 \\
141.2\end{array}$ & $\begin{array}{l}-3.36 \\
-8.43 \\
-27.5 \\
0.41 \\
-0.98 \\
-6.22\end{array}$ \\
\hline $\begin{array}{l}\mathrm{Fe} \\
\text { (II) }\end{array}$ & 2039.0 & -8441.23 & -483.5 & 322.78 & -36.23 & -145.9 & $\begin{array}{l}28.742 \\
29.045 \\
29.165 \\
28.863\end{array}$ & $\begin{array}{l}60.926 \\
50.543 \\
47.553 \\
51.339\end{array}$ & $\begin{array}{l}0.246 \\
-1.242 \\
-3.323 \\
0.007\end{array}$ & $\begin{array}{l}255.5 \\
257.4 \\
256.5 \\
254.8 \\
254.9 \\
253.8\end{array}$ & $\begin{array}{l}155.6 \\
85.97 \\
-49.92 \\
162.6 \\
167.8 \\
143.0\end{array}$ & $\begin{array}{l}-2.28 \\
-8.09 \\
-20.9 \\
0.23 \\
-1.28 \\
-5.54\end{array}$ \\
\hline Co & 2152.6 & -16502.9 & 160.06 & 322.68 & -44.73 & -193.2 & $\begin{array}{l}29.04 \\
29.16 \\
28.85\end{array}$ & $\begin{array}{l}50.45 \\
49.41 \\
50.89\end{array}$ & $\begin{array}{l}-1.564 \\
-3.965 \\
-0.24\end{array}$ & $\begin{array}{l}257.4 \\
256.5 \\
254.7 \\
254.9\end{array}$ & $\begin{array}{l}83.92 \\
-50.16 \\
161.8 \\
166.4\end{array}$ & $\begin{array}{l}-9.19 \\
-17.9 \\
-0.03 \\
-1-69\end{array}$ \\
\hline
\end{tabular}




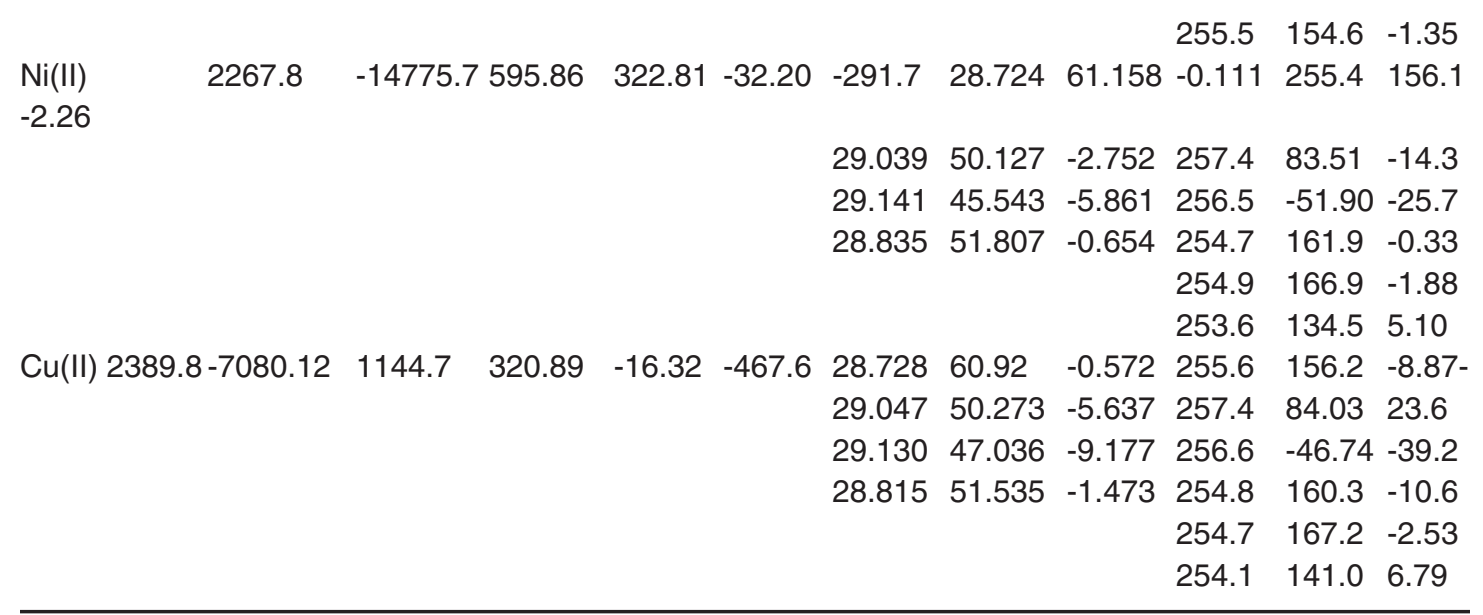

a. ADF Numbers. Dia: Diamagnetic [core \& valence tensors]; Para: Paramagnetic $\left[b^{\wedge}, u^{\wedge}, s^{\wedge}\right.$ \& gauge tensors]; SO: Spin Orbit [4 paramagnetic terms with same names but different values; may shield or deshield so can be (-) or (+)]

Table: 7. ESR and NQR Parameters of N, C and $\mathrm{H}$ from Software for $\left[\mathrm{Phen}_{2} \mathrm{M}^{\mathrm{n}+}{ }^{\mathrm{n}}\right.$ Complexes

\begin{tabular}{|c|c|c|c|c|c|}
\hline $\mathrm{M}^{\mathrm{n}+}$ & $\begin{array}{l}\mathbf{g}_{\mathrm{n}} \cdot \mathbf{A}_{\text {ten }} \text { values of } \\
4 \text { types of } \mathrm{H} \text {; each } \\
\text { type having } 4 \mathrm{H}\end{array}$ & $\begin{array}{l}g_{n} \cdot A_{\text {ten }} \text { value } \\
\text { of each one } \\
\text { of } 4 \mathrm{~N}\end{array}$ & $\begin{array}{l}g_{\mathrm{n}} \cdot \mathbf{A}_{\text {ten }} \text { values } \\
\text { of } 6 \text { types } \\
\text { of } \mathrm{C} \text {; each } \\
\text { type having } 4 \mathrm{C}\end{array}$ & $\begin{array}{l}\eta \text { values of } 6 \text { types } \\
\text { of } C \text { each type } \\
\text { having } 4 \mathrm{C}\end{array}$ & $\begin{array}{l}\text { NQCC and } \\
\eta \text { values of } \\
\text { each } N\end{array}$ \\
\hline $\mathrm{Ti}(\mathrm{III})$ & $\begin{array}{l}0.776,0.898 \\
-0.087-0.092\end{array}$ & -7.654 & $\begin{array}{c}3.109,-0.070,0.05 \\
1.994,18.360,0.197\end{array}$ & $\begin{array}{l}0.222,0.251,0.137 \\
0.411,0.680,0.336\end{array}$ & $\begin{array}{l}-1.786 \\
(0.969)\end{array}$ \\
\hline V(III) & $\begin{array}{l}0.244,0.572 \\
0.465,0.118\end{array}$ & -6.862 & $\begin{array}{r}6.118,0.057,0.863 \\
0.573,8.492,0.317\end{array}$ & $\begin{array}{l}0.394,0.267 .0 .141 \\
0.443,0.617,0.339\end{array}$ & $\begin{array}{l}1.621 \\
(0.558)\end{array}$ \\
\hline $\operatorname{Cr}(I I I)$ & $\begin{array}{l}0.115,-0.324 \\
-0.70,0.825\end{array}$ & -4.715 & $\begin{array}{l}3.261,0.059,0.08 \\
3.429,0.207,0.795\end{array}$ & $\begin{array}{l}0.345,0.250,0.139 \\
0.465,0.606,0.332\end{array}$ & $\begin{array}{l}1.511 \\
(0.464)\end{array}$ \\
\hline $\mathrm{Mn}(\mathrm{II})$ & $\begin{array}{c}-1.233,0.629 \\
-0.333,0.88\end{array}$ & 2.184 & $\begin{array}{c}1.125,-0.357,0.436 \\
0.313,3.512,0.122\end{array}$ & $\begin{array}{l}0.104,0.150,0.080 \\
0.256,0.597,0.229\end{array}$ & $\begin{array}{l}-2.112 \\
(0.635)\end{array}$ \\
\hline $\mathrm{Fe}(\mathrm{II})$ & $\begin{array}{c}-0.962,0.677 \\
0.247,0.152\end{array}$ & 5.221 & $\begin{array}{l}0.831,-0.106,0.314 \\
0.553,2.012,0.118\end{array}$ & $\begin{array}{l}0.038,0.154,0.077 \\
0.247,0.683,0.230\end{array}$ & $\begin{array}{l}-2.073 \\
(0.597)\end{array}$ \\
\hline $\mathrm{Co}(\mathrm{II})$ & $\begin{array}{c}-0.895,0.928 \\
0.913,0.299\end{array}$ & 9.623 & $\begin{array}{c}0.857,0.188,0.292 \\
0.95,1.622,0.158\end{array}$ & $\begin{array}{l}0.092,0.160,0.078 \\
0.262,0.665,0.231\end{array}$ & $\begin{array}{l}-1.893 \\
(0.698)\end{array}$ \\
\hline $\mathrm{Ni}(\mathrm{II})$ & $\begin{array}{l}-1.191,1.907 \\
2.324,0.466\end{array}$ & 16.892 & $\begin{array}{l}-2.322,0.146,0.321 \\
1.922,2.661,0.015\end{array}$ & $\begin{array}{l}0.056,0.155,0.077 \\
0.276,0.661,0.230\end{array}$ & $\begin{array}{l}-1.807 \\
(0.710)\end{array}$ \\
\hline $\mathrm{Cu}(\mathrm{II})$ & $\begin{array}{l}-1.686,3.080 \\
2.934,-0.62\end{array}$ & 19.753 & $\begin{array}{c}-2.992,0.349,0.372 \\
2.06,5.007,-0.866\end{array}$ & $\begin{array}{l}0.032,0.144,0.076 \\
0.261,0.679,0.211\end{array}$ & $\begin{array}{l}-1.959 \\
(0.581)\end{array}$ \\
\hline
\end{tabular}

the same value and third of higher value was called $\mathrm{g}_{\|}$(b) Two "a" called a $\perp$ were of the same value and the third of higher value was called $a_{11}$. (c) Two of the three q parameters were of the same value. (d) $\eta=0$. Relation (4) was applicable to all to calculate $\mathrm{H}^{\wedge}$. Individual values of these five factors in the total value of $\mathrm{H}^{\wedge}$ were given at bottom and were represented as $(\rightarrow) . \Delta \mathrm{E}_{\mathrm{hf}} \eta \eta$, Laplace equation were calculated by relations $(7,8,10)$ respectively in Tables:7-9.NQCC, a, q were expressed in $\mathrm{MHz}$ while $g$ was unit less.

Confirmation of Spatial Equivalence of N, C, H in complexes

It was confirmed by following two ways: 
From the equivalence of NMR parameters

For ascertaining the stereochemistry of the complexes, the 4 coordinating $\mathrm{N}$, the $24 \mathrm{C}$ and the $16 \mathrm{H}$ were classified according to their spatial displacements. The metal ion formed a class of its own. The spatially equivalent species possessed same values of $\delta, \sigma$ along with each one of the ten contributing terms towards the total value of $\sigma$ of the constituents. $\delta \mathrm{M}^{\mathrm{n}+}, \delta{ }^{14} \mathrm{~N}, \delta{ }^{13} \mathrm{C}, \delta{ }^{1} \mathrm{H}, \sigma \mathrm{M}^{\mathrm{n}+}$, $\sigma^{14} \mathrm{~N}, \sigma{ }^{13} \mathrm{C}, \sigma{ }^{1} \mathrm{H}$ and 10 contributing terms of all the 45 species in each one of the 8 complexes were reported(Tables 5,6). All the four coordinating ${ }^{1} \mathrm{~N}$ in each complex were spatially equivalent with the same value each for $\sigma^{14} \mathrm{~N}, \delta^{14} \mathrm{~N}$ and the 10 contributing terms respectively. Each complex having $16{ }^{1} \mathrm{H}$ contained four types of stereo chemically different ${ }^{1} \mathrm{H}$; each type possessing four equivalent protons as they showed four different series of values $\sigma^{1} \mathrm{H}, \delta^{1} \mathrm{H}$ and 10 contributing terms respectively. The $24{ }^{13} \mathrm{C}$ of each complex contained 6 types of spatially different ${ }^{13} \mathrm{C}$; each type having four equivalents ${ }^{13} \mathrm{C}$ as they gave six different series of values of $\sigma^{13} \mathrm{C}$ and $\delta^{13} \mathrm{C}$ and contributing 10 terms respectively.

From equivalence among five NMR, ESR, NQR parameters

A total of five parameters of three techniques $\left[\operatorname{ESR}\left(\mathrm{A}_{\text {ten }}\right), \operatorname{NQR}(\operatorname{NQCC}, \eta)\right.$ and $\left.\operatorname{NMR}(\sigma, \delta)\right]$ were

Table: 8. ESR and NQR Parameters ${ }^{\star}$ of $M^{+n}$ from Software* for $\left[P_{h e n} M^{M}\right]^{n+}$ Complexes

\begin{tabular}{|c|c|c|c|c|c|}
\hline Complex & g values & g n .a\& [A ten $]^{*}$ & q \& (Laplace) $)^{10}$ & NQCC\& $(\eta)^{8}$ & $\mathrm{ZFS}[\mathrm{D}, \mathrm{E}]$ \\
\hline $\mathrm{Ti}(\mathrm{III})$ & $\begin{array}{l}1.979469 \\
1.979473 \\
1.998857 \\
\mathrm{~g}_{\text {iso }} 1.985933\end{array}$ & $\begin{array}{l}-0.153030 .10^{3} \\
-0.109561 .10^{3} \\
-0.109567 .10^{3} \\
{[-124.053]}\end{array}$ & $\begin{array}{l}-0.871306 .10^{1} \\
0.435652 .10^{1} \\
0.435654 .10^{1} \\
(0.000)\end{array}$ & $\begin{array}{l}-174.261 \\
(0.000)\end{array}$ & $\begin{array}{l}{[0.000} \\
0.000]\end{array}$ \\
\hline V(III) & $\begin{array}{l}1.918096 \\
1.982626 \\
1.982627 \\
\mathrm{~g}_{\text {iso }} 1.961116\end{array}$ & $\begin{array}{l}0.139679 .10^{3} \\
0.154964 .10^{3} \\
0.154965 .10^{3} \\
{[149.869]}\end{array}$ & $\begin{array}{l}0.444424 .10^{\circ} \\
-0.222210 .10^{\circ} \\
-0.222214 .10^{\circ} \\
(0.000)\end{array}$ & $\begin{array}{l}18.666 \\
(0.0001)\end{array}$ & $\begin{array}{r}{[-4.10898} \\
-0.00000]\end{array}$ \\
\hline $\mathrm{Cr}(\mathrm{III})$ & $\begin{array}{l}1.983371 \\
1.983371 \\
2.001192 \\
\mathrm{~g}_{\text {iso }} 1.989312\end{array}$ & $\begin{array}{l}0.124604 .10^{1} \\
0.127569 .10^{2} \\
-0.127570 .10^{2} \\
{[-8.0893]}\end{array}$ & $\begin{array}{l}0.230748 .10^{1} \\
-0.115370 .10^{1} \\
-0.115378 .10^{1} \\
(0.000)\end{array}$ & $\begin{array}{l}13.845 \\
(0.000)\end{array}$ & $\begin{array}{l}{[2.703741,} \\
0.000001]\end{array}$ \\
\hline $\mathrm{Mn}(\mathrm{II})$ & $\begin{array}{c}2.000961 \\
2.002174 \\
2.002174 \\
g_{\text {iso }} 2.00177\end{array}$ & $\begin{array}{l}-0.301476 .10^{2} \\
0.117779 .10^{2} \\
-0.117776 .10^{2} \\
{[-17.9011]}\end{array}$ & $\begin{array}{l}-0.383780 .10^{1} \\
0.191890 .10^{1} \\
0.191890 .10^{1} \\
(0.000)\end{array}$ & $\begin{array}{r}-76.756 \\
(0.000)\end{array}$ & $\begin{array}{l}{[-0.00684} \\
0.000000]\end{array}$ \\
\hline $\mathrm{Co}(\mathrm{II})$ & $\begin{array}{l}2.056954 \\
2.056955 \\
2.135381 \\
g_{\text {iso }} 2.083097\end{array}$ & $\begin{array}{l}-0.384734 .10^{2} \\
0.384718 .10^{2} \\
-0.187778 .10^{2} \\
{[-31.9076]}\end{array}$ & $\begin{array}{l}-0.252450 .10^{1} \\
0.126225 .10^{1} \\
0.126225 .10^{1} \\
(0.000)\end{array}$ & $\begin{array}{l}-106.029 \\
(0.000)\end{array}$ & $\begin{array}{l}{[-6.08746,} \\
0.0000]\end{array}$ \\
\hline $\mathrm{Ni}(I I)$ & $\begin{array}{l}2.007395 \\
2.147515 \\
2.147515 \\
\mathrm{~g}_{\text {iso }} 2.10081\end{array}$ & $\begin{array}{l}0.152867 .10^{2} \\
0.152867 .10^{2} \\
-0.155095 .10^{2} \\
{[5.02128]}\end{array}$ & $\begin{array}{l}-0.551246 .10^{1} \\
0.551245 .10^{1} \\
0.110249 .10^{2} \\
(0.000)\end{array}$ & $\begin{array}{l}66.1494 \\
(0.000)\end{array}$ & $\begin{array}{l}{[24.31671,} \\
0.00000]\end{array}$ \\
\hline $\mathrm{Cu}(\mathrm{II})$ & $\begin{array}{l}2.008951 \\
2.071098 \\
2.075582 \\
g_{\text {iso }} 2.051877\end{array}$ & $\begin{array}{l}-0.350692 .10^{3} \\
0.271541 .10^{2} \\
0.273496 .10^{2} \\
{[-98.7293]}\end{array}$ & $\begin{array}{l}641568.10^{1} \\
0.320224 .10^{1} \\
-0.321343 .10^{1} \\
(0.00174))\end{array}$ & $\begin{array}{l}38.494 \\
(0.000))\end{array}$ & - \\
\hline
\end{tabular}

NQCC a, q, are expressed in $\mathrm{MHz}$ and $\mathrm{g}$ is unit less. *To be used in the calculation of $\mathrm{H}^{\wedge}$ and $\Delta \mathrm{E}_{\mathrm{hf}}$ in Table: 8. 
Table: 9. Calculation of $\mathrm{H}^{\wedge}$ and $\Delta$ Eh $\mathrm{f}$ Parameters of [Phen2 $\left.\mathrm{M}\right] \mathrm{n}+$ Complexes

\begin{tabular}{|c|c|c|c|c|c|c|}
\hline $\begin{array}{l}\mathrm{M}^{\mathrm{n}+} \\
\text { Relation }\end{array}$ & $\begin{array}{c}\text { g\& giso }\{\mathbf{g}- \\
\text { contribution }\} \\
\mathrm{MHz}\end{array}$ & $\begin{array}{c}\text { [Aten]\& } \\
\text { ( } \Delta \text { Eh f /A ten ) c } \\
\{\text { a-contribution }\} \\
\text { MHz }\end{array}$ & $\begin{array}{c}\text { NQCC }\{Q-\{ \\
\text { contribution }\} \\
\mathrm{MHz}\end{array}$ & $\left\{\begin{array}{c}\{\text { I-contribution }\} \\
\mathrm{MHz}^{*}\end{array}\right.$ & $\begin{array}{c}{[D, E](Z F S ;} \\
c m-1)(4,5) \\
\left.\{M H z\}^{* *}\right\}^{7}\end{array}$ & $\begin{array}{c}\mathrm{H}^{\wedge}(\mathrm{MHz})^{\star \star \star} \\
\{\mathrm{J} \text { mol1- }\}\end{array}$ \\
\hline $\begin{array}{l}\mathrm{Ti}(\mathrm{III}) \\
(4)\left\{\mathrm{H}^{\wedge}\right\} \\
(6)\end{array}$ & $\begin{array}{c}\| 1.998857 \\
\perp 1.979469 \\
\perp 1.979473 \\
\text { giso1.985933 } \\
(1.399 \mathrm{H} \|+2.77 \mathrm{H} \perp) \\
\{11714.87+\} \\
22970.344\end{array}$ & $\begin{array}{c}A \| 487.480 \\
A \perp 347.380 \\
A \perp 347.381 \\
{[393.330](0.88)} \\
-) \quad\{1474.988\} \\
(1474.988)\end{array}$ & $\begin{array}{l}(72.609) \\
\{72.609\}\end{array}$ & $\begin{array}{c}(-0.00060 \mathrm{HO}) \\
\quad\{-5.033\}\end{array}$ & $\begin{array}{c}{[0.000,]} \\
0.000 \\
(0.000) \\
\{0.000\}\end{array}$ & $\begin{array}{c}(36237.88) \\
\{14.464\}\end{array}$ \\
\hline $\begin{array}{l}\mathrm{V}(\mathrm{III}) \\
(6) \\
(4)\left\{\mathrm{H}^{\wedge}\right\}\end{array}$ & $\begin{array}{c}1.918096 \\
\perp 1.982627 \\
\text { giso } 1.961116 \\
(2.684 \mathrm{H} \|+5.55 \mathrm{H} \perp) \\
\{21567.062+ \\
46096.94\}\end{array}$ & $\begin{array}{c}A \| 94.951 \\
A \perp 105.342 \\
{[101.878](0.88)} \\
-) \quad(1069.719) \\
\{1069.719\}\end{array}$ & $\begin{array}{l}(-32.666) \\
\{-32.666\}\end{array}$ & $\begin{array}{c}(0.003952 \mathrm{HO}) \\
\quad\{33.150\}\end{array}$ & $\begin{array}{c}{[-4.10898} \\
-0.0000] \\
(-5.4786) \\
\{164245 \\
495\}\end{array}$ & $\begin{array}{c}(232979.70) \\
\{92.966\}\end{array}$ \\
\hline $\begin{array}{l}\mathrm{Cr}(\mathrm{III}) \\
\left.(4)\left\{\mathrm{H}^{\wedge}\right\}\right) \\
(6\end{array}$ & $\begin{array}{c}\| 2.000961 \\
1.983371 \\
\perp 1.983371 \\
\text { giso } 1.989312 \\
(4.20 \mathrm{H} \|+8.327 \mathrm{H} \perp) \\
\{35210.824+ \\
69187.98\}\end{array}$ & $\begin{array}{c}A \| 3.939 \\
A \perp-40.324 \\
A \perp-40.324 \\
{[25.570](0.81)} \\
\quad(-172.598) \\
\{-172.598\}\end{array}$ & 13.845 & $\begin{array}{c}(-0.00036 \mathrm{HO}) \\
\{-3.020\}\end{array}$ & $\begin{array}{c}(6.7594) \\
\{202640.29\}\end{array}$ & $\begin{array}{c}(306866.69) \\
\{122.449\}\end{array}$ \\
\hline $\begin{array}{l}\operatorname{Mn}(I I I) \\
(4)\left\{H^{\wedge}\right\} \\
\text { (6) }\end{array}$ & $\begin{array}{c}\| 2.000961 \\
\perp 2.002174 \\
\perp 2.002174 \\
\text { giso } 2.00177 \\
(7.00 \mathrm{H} \|+14.01 \perp) \\
\{58677.93+ \\
117510.884\}\end{array}$ & $\begin{array}{c}A \|-21.728 \\
A \perp-8.489 \\
A \perp-8.489 \\
{[-12.902](0.96)} \\
(-241.913) \\
\{-241.913\}\end{array}$ & $\begin{array}{l}(31.982) \\
\{31.982\}\end{array}$ & $\begin{array}{c}((0.001889 \mathrm{H} 0) \\
\{15.454\}\end{array}$ & $\begin{array}{c}(-0.05984) \\
\{1794.198\}\end{array}$ & $\begin{array}{c}(177788.54) \\
\{70.943\}\end{array}$ \\
\hline $\begin{array}{l}\mathrm{Co}(\mathrm{III}) \\
(4)\left\{\mathrm{H}^{\wedge}\right\} \\
\text { (6) }\end{array}$ & $\begin{array}{c}\| 2.135381 \\
\perp 2.056954 \\
\perp 2.056955 \\
\text { giso } 2.083097 \\
(5.482 \mathrm{H} \|+8.636 \mathrm{H} \perp) \\
\{49040.235+ \\
74417.584\}\end{array}$ & $\begin{array}{c}A \|-14.204 \\
A \perp-29.100 \\
A \perp-29.102 \\
{[-24.135](0.90)} \\
\text { L) } \quad(-380.126) \\
\quad\{-380.126\}\end{array}$ & $\begin{array}{l}(185.551) \\
\{188.551\}\end{array}$ & $\begin{array}{c}(0.00353 \mathrm{H} 0) \\
\quad\{29.611\}\end{array}$ & $\begin{array}{c}(-15.2187) \\
\{456243 . \\
649\}\end{array}$ & $\begin{array}{c}(579539.50) \\
\{231.254\}\end{array}$ \\
\hline $\begin{array}{l}\mathrm{Ni}(\mathrm{III}) \\
(4)\left\{\mathrm{H}^{\wedge}\right\} \\
(6)\end{array}$ & $\begin{array}{c}\| 2.007395 \\
\perp 2.147515 \\
\perp 2.147515 \\
\text { giso } 2.10081 \\
(2.809 \mathrm{HII}+6.01 \mathrm{H} \perp) \\
\{23622.329+ \\
54069.060\}\end{array}$ & $\begin{array}{c}A \| 49.025 \\
A \perp-48.321 \\
A \perp-48.321 \\
{[-15.872](2.65)} \\
\quad(-71.424) \\
\{-71.424\}\end{array}$ & $\begin{array}{l}(16.537) \\
\{16.537\}\end{array}$ & $\begin{array}{c}(-0.00057 \mathrm{HO}) \\
\{-4.781\}\end{array}$ & $\begin{array}{c}(32.422) \\
\{971995.502\}\end{array}$ & $\begin{array}{c}(1049627.2) \\
\{418.834\}\end{array}$ \\
\hline $\begin{array}{l}\mathrm{Cu}(\mathrm{III}) \\
(4)\left\{\mathrm{H}^{\wedge}\right\} \\
\text { (6) }\end{array}$ & 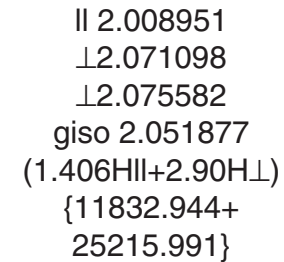 & $\begin{array}{c}A \| 236.613 \\
A \perp-18.321 \\
A \perp-18.452 \\
{[66.610](1.79} \\
(149.873) \\
\{149.873\}\end{array}$ & 9 ) $\begin{array}{l}(9.624) \\
\{9.624\}\end{array}$ & $\begin{array}{c}(-0.001695 \mathrm{HO}) \\
\{-14.218\}\end{array}$ & $\begin{array}{l}(0.000) \\
\{0.000\}\end{array}$ & $\begin{array}{c}(37222.65) \\
\{14.857\}\end{array}$ \\
\hline
\end{tabular}


Table: 10. Designations of IR Active Bands in [Phen2 M] $\mathrm{n}+$ Complexes

\begin{tabular}{llll}
\hline Complex with $\mathbf{M}^{+\mathbf{n}}$ & $\begin{array}{l}\text { Vibration Symmetry } \\
\text { of bands }\end{array}$ & $\begin{array}{l}\text { IR active } \\
\text { bands }\end{array}$ & $\begin{array}{l}\text { Vibration Symmetry } \\
\text { Class }\end{array}$ \\
\hline $\begin{array}{l}\mathrm{Ti}(\mathrm{III}), \mathrm{V}(\mathrm{III}), \mathrm{Cr}(\mathrm{III}), \mathrm{Mn}(\mathrm{II}), \\
\mathrm{Fe}(\mathrm{II}), \mathrm{Co}(\mathrm{II}), \mathrm{Ni}(\mathrm{II}), \mathrm{Cu}(\mathrm{II})\end{array}$ & $\mathrm{A}(129)$ & $\mathrm{A}(129)$ & {$[129 \mathrm{~A}]$} \\
\hline
\end{tabular}

*Numbers in parentheses indicate bands of a specific symmetry

Table: 11. $\sigma \mathrm{N}$ values of 1, 10-Phenanthroline and its complexes with metal ions

\begin{tabular}{llllllllll}
\hline$\sigma$ values & Phen & $\mathrm{Ti}(\mathrm{III})$ & $\mathrm{V}$ (III) & $\mathrm{Cr}(\mathrm{III})$ & $\mathrm{Mn}$ (II) & $\mathrm{Fe}(\mathrm{II})$ & $\mathrm{Co}(\mathrm{II})$ & $\mathrm{Ni}(\mathrm{II})$ & $\mathrm{Cu}(\mathrm{II})$ \\
\hline$\sigma \mathrm{N}$ & -138.4 & 316.50 & 296.30 & 310.20 & 178.39 & 140.68 & 84.80 & -1.15 & -163.1 \\
\hline
\end{tabular}

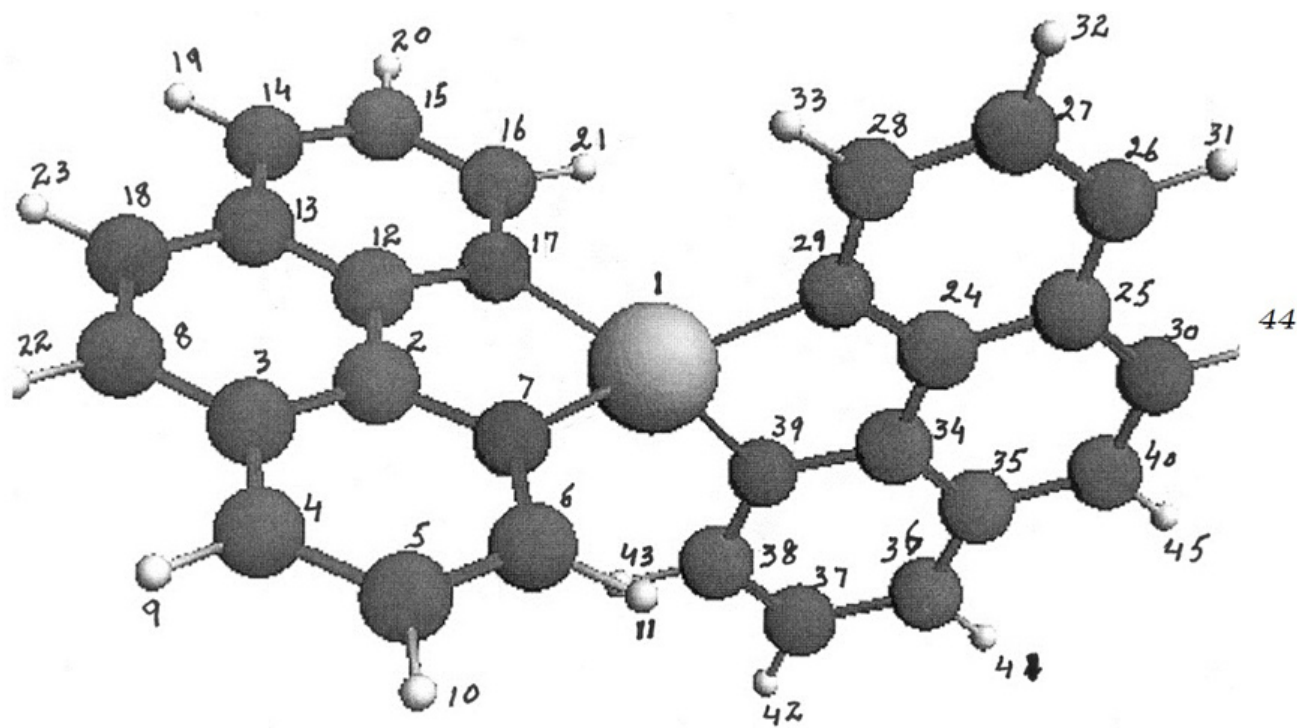

Fig.1: [Phen $\left.{ }_{2} \mathrm{M}\right]^{\mathrm{n}+}\{\mathrm{M}=\mathrm{Ti}(\mathrm{III}), \mathrm{V}(\mathrm{III}), \mathrm{Cr}(\mathrm{III}), \mathrm{Mn}$ (II), $\mathrm{Fe}(\mathrm{II}), \mathrm{Co}(\mathrm{II}), \mathrm{Ni}(\mathrm{II}), \mathrm{Cu}(\mathrm{II})$ at:1 $\}$

used to ascertain similar stereochemistry of 8 paramagnetic complexes. Four stereo chemically equivalent $\mathrm{N}$ possessed the same values of above named five parameters. The four parameters: $g_{n} \cdot A_{\text {ten }}, \eta, \sigma, \delta$ of $24{ }^{13} \mathrm{C}$ were categorized into 6 types(NQCC $=0$ for all); each having 4 equivalents C. Three parameters $\left(g_{n} \cdot A_{\text {ten }}, \sigma, \delta\right)$ of the $16 \mathrm{H}$ were of 4 types (NQCC and $\eta=0$ for all); each type having 4 equivalents ${ }^{1} \mathrm{H}$. So the three techniques corroborated to confirm that each one of these complexes possess one type of $4 \mathrm{~N}$; six types of $24 \mathrm{C}$ and four types of $16 \mathrm{H}$. Further, the two NMR parameters $(\sigma, \delta)$ of constituents were shown in Table: 5 and the remaining three [one of EPR $\left(A_{\text {ten }}\right)$ and two of NQR (NQCC, $\eta$ ) were shown in (Table: 7$)$. Again, with $\mathrm{l}=1 / 2$ for ${ }^{1} \mathrm{H}$ and ${ }^{13} \mathrm{C}$; their $\mathrm{NQCC}=0.0$. This left 4 parameters each for ${ }^{1} \mathrm{H}$ and ${ }^{13} \mathrm{C}$. All the $\eta$ values were zero for all the $16^{1} \mathrm{H}$.So three parameters were reported for ${ }^{1} \mathrm{H}$.

\section{Evidence of MLCT Phenomenon from NMR parameters of complexes}

MLCT phenomenon would increase an electron density on the ligand by shifting electronic charge from the filled molecular orbitals of complex lying mainly on metal to its vacant molecular orbitals with energies comparable to the ligand. As $\sigma$ of a nucleus was directly related to its electron density, any change in its value should serve as an indicator to the change in electron density on it. In 7 of the 8 complexes $\left\{\right.$ except in $\left.\left[\mathrm{Phen}_{2} \mathrm{Cu}\right]^{2+}\right\}$, total shielding tensors of the $\sigma^{14} \mathrm{~N}, \sigma^{13} \mathrm{C}, \sigma^{1} \mathrm{H}$ (Tables:11,5) were 
observed to be higher and the $\delta^{14} \mathrm{~N}, \delta^{14} \mathrm{C}, \delta^{1} \mathrm{H}$ were found to be lower than their values in the uncoordinated ligand (Table:4).It confirmed the transfer of electronic charge from metal to the ligand and thereby the MLCT.

\section{IR Parameters of complexes}

The software gave values of frequencies, dipole strengths and absorption intensities of IRactive normal modes of all the 129 fundamental vibration bands of the complexes; each having $A$ (singly degenerate ) symmetry according to their IRactivities $^{48}$ with a Vibration Symmetry Class $\{129 A\}$ (Table: 10).

\section{CONCLUSIONS}

- With higher $\sigma^{14} \mathrm{~N}, \sigma^{13} \mathrm{C}, \sigma^{1} \mathrm{H}$ and lower $\delta^{14} \mathrm{~N}$, $\delta^{14} \mathrm{C}, \delta^{1} \mathrm{H}$ values of constituents in these complexes relative to uncoordinated ligand would confirm an increase in the electron density on the ligand in the complexes to lend support to the transfer of electron cloud from the metal into the ligand orbitals as per MLCT definition.

NMR, ESR and NQR results corroborated with one another to prove that all these 8 complexes possessed the same stereochemistry as all the 44 atoms were occupying the same relative positions around each one of metal ion.

\section{REFERENCES}

1. Satar, A.; Jabbar, A,; Rzokee, Ahmad, A. Int. J. Basic App. Chem. Sci. 2014, 4, 37-50.

2. Zalis, S.; Consani, C.; El Nahhas, A.; Canizzo, A.; Chergui, M.; Hart, F.; Vlecek, A. Inorg. Chim. Acta. 2011, 374, 578- 85

3. Jesse, V. G.; Christopher, J. E.; Lev, N. Z.; Mattew, E. C.; Michael, M.H.;

Darren, W. J. Chem. Sci. 2014, 5, 28992905.

4. Yutaka, T.; Obara, S.; Ogawa, S.; Nozaki, K.; Ikeda, N.; Ohno, T.; Ishii, Y. Sakai, K.; Aski, M. H. Inorg Chem. 2005, 44, 4737-46.

5. Mudasira, K. W.; Daryono, H. T.; Naoki, Y.; Hidenari, I. Z. Naturforsch. 2004, 59, 31018.

6. Sreekanth, B.; Krishnamurthy, G.; Bhojya N. H. S.; Vishnuvardhan, T. K.; Shashikumar, N. D.; Lokesh, M.R. J. Chem. Pharm. Res. 2011 , 3 , 407-19.

7. Sreekanth, B.; Krishnamurthy, G.; Naik, H.S.; Vishnuvardhan, T.K.; Vinaykumar, B. S. N. Nucleosides Nucleotides Nuc. Acids. 2011, 30, 83-96.

8. Suescun, L.; Mombru, A.; Mariezcurrena, R.A.; Baggio, R. Acta. Cryst. C. 2000, 56, 179-81.

9. Scaringe, R.; Singh, P,; Eckberg, R.P.; Hatfield, W.E.; Hodson, D.J. Inorganic Chem. 1975, 14, 1127-33.

10. Yesilel, O.; Olmez, H. J. Thermal Analy. Calorimet. 2006, 86, DOI: 10.1007/s10973-

\section{5-7137-2}

11. Chis, V.; Droghetti, A.; Isai, R.; Morari, C., Rungger, I.; Sanvito, S. AIP Conf. Proc. 2013, 57; 25-27.

12. Ozel, A.E.; Kecel, S.; Akyuz,S. Mol.Str. 2007, 834, 548-54.

13. Morigakii, M.K.; Elias, M. D.S.; Carlos, V.P. D.M.; Jamile, R.P.; Renzo, C.S.; Armando, B.; Jair, C.C.F.; Gilson, H.M.D. Quim.Nova 2009, 32.

14. Chiniforoshan, H.Z.; Sadeghian, L.; Tabrizia, H.; Tavakol, M.; Sabzalian, G.; Mohammad nezhad, H.; Goris, W.P. Mol. Str. 2015, 1081, 237-43.

15. Sanotra, S.; Gupta, R.; Gupta, U.; Khajuria, Y. Spectrochim. Acta. Part-A. 2014, 129, 39299.

16. Euan, K. B.; Lucia, C.; Ulli, E.; Ludovica, M.; Guido, P.; Calogero, P.; Alessanodro, P. Inorg. Chim. Acta. 2008, 361, 2375- 84.

17. Panina, N.S.; Demidov, V. N.; Simanova, S. A. Russian J. Gen. Chem. 2008, 78, 919-24.

18. Zn, B.; Xiao, Y. Prog. zeolite microporous mat. PTC a-c. 1997, 105, 615-22.

19. Kaupp, M.; Malkin, V.G.; Malkina, O.L. Encyclo. Computat. Chem. 1998, 1857-66.

20. Schreekenbatch, G.; Zieglar, T. Theo. Chem. Acc. 1998, 99, 71-78.

21. M. Buhl, M. Kaupp, O.G. Malkina, V.G. Malkin, "The DFT route to NMR chemical shifts," J. Comput.Chem. 1999,20(1), 91-105 
22. Autschbach, J.; Zieglar, T. Coord. Chem. Rev. 2003, 238/239, 83-126.

23. Buhl, M. Theo. App. 2004, 421-31.

24. Autschbach, J.; Zieglar, T. Encyclo Nuc. Mag. Reson. 2002, 9, 306-23.

25. Buhl, M.; Mauschick, F.T. Phys. Chem. Chem. Phys. 2002, 4, 5508-14.

26. Brahham, S.; Sachin, H.P.; Shivshankar, S.A.; Narsimhamurthy, T.; Rathore, R. M. Acta Crys. C. 2008, 64, $140-143$.

27. Schilt, A. A.; Taylor, R. C. J. Inorg. Nuc. Chem. 1959, 9, 211-21.

28. Sehgal, M.L.; Ahmad, I. Oriental J. Chem. 2017, 33.

29. Sharma, S.; Chander, S.; Sehgal, M.L.; Ahmad, I. Oriental J. Chem. 2015, 31, 141727.

30. Singh, H.; Bhardwaj, A.K.; Sehgal, M.L.; Ahmad, I. Oriental J. Chem. 2015, 31, 67179.

31. Singh, H.; Bhardwaj, A.K.; Sehgal, M.L.; Javed, M.; Ahmad, I. Oriental J. Chem. 2015, 31, 1461-68.

32. Schreckenbach, G.; Ziegler, T. J. Phys. Chem. 1995, 99, 606-11.

33. Schreckenbach, G.; Ziegler, T. Int. J.Quantum Chem. 1997, 61, 899-918.

34. Wolff, S.K.; Ziegler, T. J. Chem. Phys. 1998, 109, 895.
35. Singh, H.; Bhardwaj, A.K.; Sehgal, M.L.; Susheel, K.M. Int. J. Curr. Res. Rev. 2012, 4, 12-28.

36. Singh, H.; Bhardwaj, A.K.; Sehgal, M.L.; Susheel, K.M. Int. J. Curr. Res. Rev. 2013, 5, 13-31.

37. Singh, H.; Bhardwaj, A.K.; Sehgal, M.L.; Susheel, K.M. Int. J. Curr. Res. Rev. 2013, 5, 71-88.

38. Neese, F. Chem. Phys. 2007, 127, 164112.

39. Schmitt, S.; Jost, P.; Van Wullen, C. J. Chem. Phys. 2011, 134, 194113.

40. Autschbach, J. Str. Bond. 2004, 112, 1-43.

41. Russel, D.S. Phy. methods in Chem. 1977, 227, 361-97.

42. Sandberg, K.A. Ph.D.Thesis: Chemistry Department, NC State University,1998.

43. Abragam, A.;Bleaney, B.Electron Paramagnetic Res. Trans. Ions. 1986.

44. Schweiger, A.; Jeschke, G. Prin. Pulse Elec. Paramagnetic Res. 2001

45. Weil, J. A.; Olton, J.R. Elec. Paramagnetic Res. Spectro. Elem. Theo. Applicat. 2007.

46. Edmonds, D.T.; et al. Adv. Quadrup. Res. 1974, 1, 145.

47. Smith, A. S. Adv. Nuc. Quadrup. Res. 1975, 2, 1977, 3.

48. Nakamoto, K. Infrared Raman. Spec. Inorg. Coord. Comp. 1986, 291-98. 